\title{
Método de Penman-Monteith e Hargreaves-Samani para obtenção da ETo em ano chuvoso e seco
}

\section{Comparison between Penman-Monteith and Hargreaves-Samani to obtain ETo in rainy and dry years}

\author{
Erllan Tavares Costa Leitão ${ }^{1 *}$, Michael Pratini Silva de Souza ${ }^{2}$, Josivalter Araujo de Farias ${ }^{3}$, Ana Beatriz \\ Alves Araujo ${ }^{4}$, Poliana Maria da Costa Bandeira ${ }^{5}$, Priscila Pascali da Costa Bandeira 6
}

\begin{abstract}
${ }^{1}$ Engenheiro agrônomo, Mestre em Manejo de Solo e Água, Universidade Federal Rural do Semi-Árido, Mossoró, +55 84 998592545; erllantavares@ hotmail.com; ${ }^{2}$ Biólogo, Mestre em Ecologia e Conservação, Universidade Federal Rural do Semi-Árido, Mossoró; michaelpratini@ @otmail.com; ${ }^{3}$ Engenheiro agrônomo, Mestrando em Agronomia/Fitotecnia, Universidade Federal do Ceará, Fortaleza, josivalter_araujo@ hotmail.com; ${ }^{4}$ Engenheira agrícola e ambiental, Doutora em Manejo de Solo e Água, Universidade Federal Rural do Semi-Árido, Mossoró; beatrizufersa@ gmail.com; ${ }^{5}$ Engenheira agrícola e ambiental, Mestranda em Engenheira Agrícola, Universidade Federal de Viçosa, polianabandeira1@hotmail.com; ${ }^{6}$ Engenheira agrícola e ambiental, Universidade Federal Rural do Semi-Árido, Mossoró; priscilabandeira_@outlook.com
\end{abstract}

\section{A R T I G O \\ Recebido: 04/07/2019 \\ Aprovado: 24/09/2020}

Palavras-chave:

Evapotranspiração

Variáveis climáticas

Semiárido
Key words:

Evapotranspiration

Climatic variables

Brazilian semiarid

\section{R E S U M O}

O planejamento e aproveitamento da água na produção agrícola, bem como o desenvolvimento de metodologias que permitam estimar o correto volume de água são fundamentais para o estabelecimento de planos eficientes de irrigação, evitando o desperdício. A evapotranspiração está diretamente relacionada ao consumo de água das plantas, desta forma, foram desenvolvidos diversos métodos para a determinação da evapotranspiração utilizando para isso, as variáveis meteorológicas. Dessa forma objetivou-se avaliar umas das metodologias mais utilizadas, que é o conhecida como método de Hargreaves-Samani para estimar a evapotranspiração de referência (ETo) em condições de baixa e elevada pluviosidade, utilizando como referência o método de Penman-Monteith-FAO56. Foram empregados dados de uma estação meteorológica automática pertencente à Universidade Federal Rural do Semi-Árido, em Mossoró (RN), os dados mencionados são referentes ao período de janeiro de 2011 a dezembro de 2012. A ETo foi estimada sendo considerada de uso prático, o modelo de Hargreaves-Samani que utiliza elementos meteorológicos de fácil obtenção como a temperatura, umidade relativa do are velocidade do vento, permitindo a estimativa da ETo é considerado de uso prático. Verificou-se que, as estimativas a partir do método de HargreavesSamani superestimaram os valores de ETo quando comparado com o método padrão em condições de elevada umidade para o ano de 2011, além de apresentar significância quanto a variável velocidade do vento, desta forma podendo ser fortemente influenciada por ela. Diante disso verificase a necessidade de calibração ou parametrização dos coeficientes da equação de HargreavesSamani como alternativa para uma melhor estimativa, principalmente para cidades com influência litorânea.

\section{A B S T R A C T}

The planning and use of water in agricultural production, as well as the development of methodologies wich allow estimating the correct volume of water are essential for the establishment of efficient irrigation plans, avoiding waste in periods of water deficit. Evapotranspiration is directly related to the water consumption of plants, thus several methods were developed to determine the evapotranspiration used for this, the meteorological variables. Thus, the objective was to evaluate one of the most used methodologies, the Hargreaves-Samani method to estimate the ETo reference evapotranspiration in low and high rainfall conditions, using the Penman-Monteith method - FAO56 as reference. Automatic meteorological system, belonging to the Federal Rural University of SemiArid, in Mossoró, Rio Grande do Norte, was used. The data mentioned are for the period from January 2011 to December 2012. Considered to be practical, the Hargreaves-Samani model uses

\footnotetext{
Revista Verde

ISSN 1981-8203

Pombal, Paraíba, Brasil
}

v. 15, n.4, out.-dez., p.398-403, 2020 doi: $10.18378 /$ rvads.v15i4.7500 
meteorological elements that are easily obtained, such as temperature, relative humidity and wind speed, allowing the estimation of reference evapotranspiration. It appears that the estimates from Hargreaves-Samani overestimated the ETo values when compared to the standard method in conditions of high humidity (2011), in addition to presenting significance regarding the variable wind speed, thus being able to be strongly influenced for it. We then see the need for calibration or parameterization of the Hargreaves-Samani equation's coefficients as an alternative for a better estimate, especially for cities with coastal influence.

\section{INTRODUÇÃO}

Em cultivos agrícolas, o desperdício de água pode ocorrer através dos mecanismos de evapotranspiração, nos quais acontece de forma simultânea os processos de transpiração da planta e evaporação do solo. Na transpiração, a planta libera água em forma de vapor para a atmosfera, enquanto a evaporação é a translocação da água presente no solo para a atmosfera.

A evapotranspiração é um dos fatores mais importantes no ciclo hidrológico, baseado na ligação entre energia, clima e disponibilidade hídrica, podendo ser estimada por diferentes métodos indiretos ou medida em condições locais por meio de lisimetria (BARBIERI et al., 2020).

Diversos são os métodos de estimativa da ETo, sendo os que utilizam como base a temperatura, mais comumente aplicados nesses estudos, tendo como exemplo o método de Hargreaves-Samani. Esse método, entretanto, apresenta uma base empírica, necessitando muitas das vezes, de calibração de acordo com o local ao qual será adotado (CONCEIÇÃO, 2019).

A água necessária para a produção agrícola deve ser utilizada da forma mais racional possível, por se mostrar fator primordial para as culturas e um dos principais parâmetros para o correto planejamento, dimensionamento e manejo de qualquer sistema de irrigação (FERNANDES; NANTES, 2019).

Assim diversos pesquisadores em todo o mundo propuseram modelos indiretos para a estimativa da ETo, com diferentes concepções e variáveis envolvidas. Antes de se eleger o modelo a ser utilizado para a estimativa da ETo, é necessário saber quais as variáveis meteorológicas disponíveis e só a partir daí, verifica-se quais podem ser aplicadas, uma vez que a utilização dos diferentes métodos para certo local de interesse fica na dependência dessas variáveis (FERREIRA et al., 2019).

Muitos modelos propostos para a estimativa da ETo não podem ser aplicados em cenários diferentes daqueles em que foram originalmente desenvolvidos. A fim de resolver esse problema a Organização das Nações Unidas para Alimentação e Agricultura (FAO) padronizou o modelo de Penman-Monteith (PM) (TURCO, 2019), criando o modelo Penman-MonteithFAO56 (PM-FAO) específico para a estimativa da ETo. PMFAO é uma equação que expressa a evapotranspiração de uma cultura hipotética de referência com uma altura uniforme de 0,12 $\mathrm{m}$, a resistência de superfície fixa de $70 \mathrm{~s} \mathrm{~m}^{-1}$ e um albedo de 0,23 , crescendo ativamente, cobrindo completamente o solo e sem sofrer estresse hídrico. Nessas condições, os únicos fatores que afetam ETosão as variáveis meteorológicas, consequentemente, a ETo pode ser considerada uma variável atmosférica e pode ser estimada a partir de dados meteorológicos (SENTELHAS et al., 2010; de LIMA JUNIOR, et. al., 2016).

O processo de estimativa exige medições confiáveis de elementos meteorológicos tais como: temperatura do ar, umidade relativa do ar, radiação solar e velocidade do vento. Porém, frequentemente nem todos esses elementos estão disponíveis, em razão do número limitado de estações meteorológicas devidamente equipadas, ou apresentam grandes falhas e/ou imprecisões nas medições, por estas razões, este método tem o seu uso limitado pela falta de elementos meteorológicos (FERNANDES et al., 2012).

Segundo Venancio et al. (2019), o uso da equação de Hargreaves-Samani (1985) é uma alternativa viável para a estimativa da ETo quando há falta de elementos meteorológicos requeridos pelo modelo PM-FAO56. O modelo de HargreavesSamani (HS) necessita apenas das temperaturas mínima, máxima e média do ar. Esse modelo vem sendo utilizado amplamente devido a sua simplicidade e a potencialidade para calibração de seus parâmetros (VENANCIO et al., 2019). Esse método pode fornecer dados confiáveis de ETo para o intervalo de cinco dias ou períodos mais longos (THEPADIA; MARTINEZ, 2012).

Como ponto limitante no processo de obtenção de dados de evapotranspiração por lisímetros destaca-se o fato de geralmente ser bastante complexo e oneroso, de forma que a obtenção de medidas está restrita a institutos de pesquisa, tendo em vista os altos custos para implantação de técnicas micrometeorológicas e lisimétricas (SILVA, 2019).

A FAO recomenda que os métodos empíricos devem ser calibrados e validados com base na equação de PenmanMonteith-FAO56 como referência (ALLEN et al., 1998).Hargreaves-Samani (1985) recomendam uma equação simples para estimar a ETo utilizando a diferença de temperatura, ela tem possibilitado estimativas adequadas da ETo em variadas partes do planeta. Portanto, objetivou-se com este trabalho comparar os valores de ETo obtidos no método de PenmanMonteith-FAO56 com Hargreaves-Samani, com finalidade de constatar a possibilidade de estimar a evapotranspiração de referência para as condições climáticas do município de Mossoró no estado do Rio Grande do Norte (RN), e assim fazer a calibração local dessa equação, para períodos chuvosos e secos.

\section{MATERIAL E MÉTODOS}

Os dados utilizados neste estudo foram oriundos da estação meteorológica automática pertencente à Universidade Federal Rural do Semi-Árido (UFERSA), em Mossoró (RN), cujas coordenadas geográficas são: latitude $5^{\circ} 12^{\prime} 48^{\prime}$ ' S, longitude $37^{\circ} 18^{\prime} 44^{\prime}$ 'W e altitude de $37 \mathrm{~m}$. Segundo a classificação climática de Köppen, o clima na região é do tipo BSwh', ou seja, quente e seco, com estação chuvosa no verão atrasando-se para o outono (CARMO FILHO et al., 1987). Mossoró se encontra aproximadamente a $30 \mathrm{~km}$ do município de Arei Branca, pertencente a Costa Branca da Costa Semiárida Brasileira(DINIZ; OLIVEIRA, 2016), sofrendo influências por proximidade com o litoral. Os dados utilizados foram obtidos no período de 01 de janeiro de 2011 a 31 de dezembro de 2012 tendo 
valores diários de temperatura do ar, radiação solar, umidade relativa do ar, pressão atmosférica e velocidade do vento sendo também essas variáveis objeto de estudo.

Para o tratamento dos dados, foi utilizado o software Excel ${ }^{\circledR}$ bem como para a montagem das equações, já para a ocasião do tratamento estatístico não paramétrico, foi utilizado o programa R Studio versão 3.4.3, que é um software livre de código aberto (R CORE TEAM, 2017). Os métodos de Penman-MonteithFAO56 (Equação 1) e de Hargreaves-Samani (Equação 2) estão descritos.

$$
\begin{array}{cl}
\text { ETo }=\frac{0,408 \Delta\left(R_{n}-G\right)+\gamma \frac{900}{T_{m e d}+273} u_{2}\left(e_{s}-e_{a}\right)}{\Delta+\gamma\left(1+0,34 u_{2}\right)} & \text { Eq. } 1 \\
\text { ETo }=0,0135 K_{T}(T+17,78)\left(T_{M A X}-T_{M I N}\right) & \text { Eq. } 2
\end{array}
$$

Em que:ETo é a evapotranspiração de referência em $\mathrm{mm} \mathrm{d}^{-1} ; \mathrm{R}_{\mathrm{n}}$ é a radiação líquida em $\mathrm{MJ} \mathrm{m}^{-2} \mathrm{dia}^{-1}$; $\mathrm{G}$ é a densidade do fluxo de calor no solo em $\mathrm{MJ} \mathrm{m}^{-2} \mathrm{dia}^{-1} ; \mathrm{T}_{\text {med }}$ é a temperatura média diária do ar em ${ }^{\circ} \mathrm{C} ; \mathrm{V}_{2}$ é a velocidade do vento média diária a $2 \mathrm{~m}$ de altura em $\mathrm{m} \mathrm{s}^{-1}$; $\mathrm{e}_{\mathrm{s}}$ é a pressão de saturação de vapor d'água em $\mathrm{kPa} ; \mathrm{e}_{\mathrm{a}}$ é a pressão atual de vapor d'água, $\mathrm{kPa} ; \Delta$ declividade da curva de pressão de vapor d'água em $\mathrm{kPa} \mathrm{C}^{-1}$, U $\gamma$ é o coeficiente psicrométrico $(\mathrm{MJ} / \mathrm{kg}) ; \lambda$ é o Calor latente de vaporização $\left(\mathrm{kPa} /{ }^{\circ} \mathrm{C}\right)$.

\section{RESULTADOS E DISCUSSÃO}

Ao se estimar a ETo na região de Mossoró pela diferença entre a ETo mensal utilizando os modelos das equações 1 e 2,observou-se variação entre os meses, mas uma variação expressiva entre os métodos de estimativa da ETo, apresentando maior variação o método de $\mathrm{HS}\left(\mathrm{R}^{2}=0,23\right)$ no ano de 2011 (Figura1) que foi o ano com maior índice pluviométrico. $\mathrm{O}$ método HS superestimou os dados, apresentando uma menor confiabilidade.

Corroborando com os dados encontrados por Nicodem e Minuzzi (2020), que ao comparar diferentes municípios, obteve clara tendência do método de HS em superestimar a ETo e quando comparado ao PM-FAO56 do que observou-se o inverso para a ETo analisada. Também foi observado que geralmente HS superestima a ETo em regiões de clima úmido, concordando com os dados em Santa Catarina e também nas regiões úmidas do Irã encontradas por Tabari et al. (2013) com superestimativas de $0,32 \mathrm{~mm} \cdot \mathrm{d}^{-1}$ a $0,96 \mathrm{~mm} \cdot \mathrm{d}^{-1}$. A ETo por HS apontou tendência de superestimar nas regiões interioranas e subestimar nas áreas costeiras da Tunísia, se comparado com os valores de ETo por PM-FAO56.

Figura 1. Distribuição da ETo estimado pelos métodos de Penman-Monteith-FAO56 e de Hargreaves-Samani em Mossoró, Rio Grande do Norte (PM-FAO 56=Penman-Monteith-FAO56)

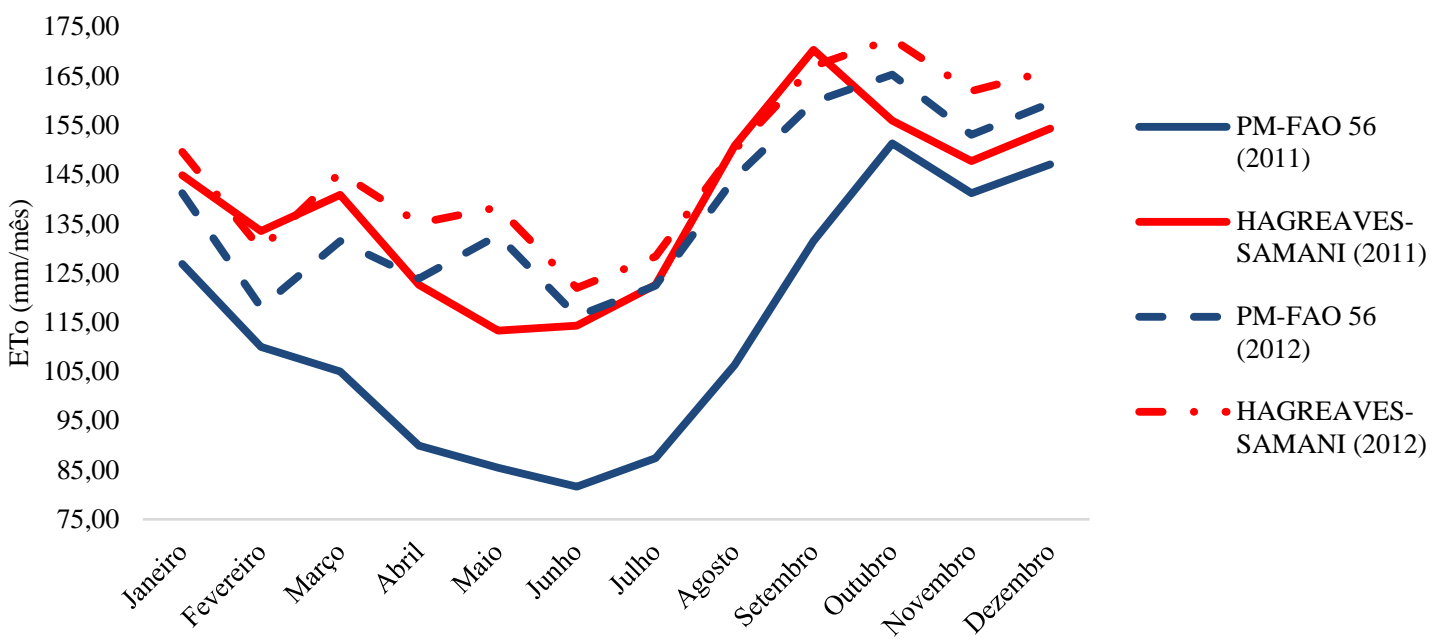

Quando avaliados a pluviosidade e ETo em ambas as equações, ao se calcular a ETo percebe-se a correlação com a pluviosidade, o ano de 2011 apresentou uma maior pluviosidade anual (Tabela 1) e consigo foram também as estimativas da ETo, com destaque para o método HS com uma eventual tendência de subestimação impulsionada pela maior pluviosidade obtida na época.

Discordando com dados obtidos por Cavalcante Junior e tal. (2011), que encontrou resultados apontando o método de Hargreaves-Samani com uma melhora significativa de seus dados quando empregados em períodos chuvosos. Assim como nos dados expostos por Lima Junior et. al (2016) que ao estudar a parametrização do modelo Hargreaves-Samani constatou que em praticamente todas as estações a equação original HS se aproximou a ETo calculada por PM-FAO56 nos primeiros meses do ano, período esse que apresenta maiores quantidades de chuvas e menores amplitudes térmicas.

As velocidades do vento em média e máxima para os anos de 2011 foram de 1,8 e 3,7 $\mathrm{m} \mathrm{s}^{-1}$, já em 2012 atingiram 2,7 e 3,9 $\mathrm{m} \mathrm{s}^{-1}$ característico de períodos mais secos e quentes, por conseguinte maior movimentação do ar. Normalmente quando utilizado o modelo de HS em regiões com valores de velocidade do vento maiores que dois metros por segundo tem necessidade do uso de coeficientes ajustados em vez do original (FOOLADMAND; HAGHIGHAT,2007). 
Tabela 1. Dados para estimativas da Evapotranspiração (ETo) pelos métodos Penman-Monteith-FAO56 e de Hargreaves-Samani ao longo dos meses nos anos de 2011 e 2012

\begin{tabular}{lllllllllllllll}
\hline & ANO & JAN & FEV & MAR & ABR & MAI & JUN & JUL & AGO & SET & OUT & NOV & DEZ & MÉDIA \\
\hline T* & 2011 & 27,33 & 27,16 & 26,61 & 26,09 & 25,86 & 25,87 & 25,51 & 26,31 & 27,71 & 27,79 & 28,05 & 28,05 & 26,86 \\
& 2012 & 28,07 & 27,39 & 28,01 & 28,38 & 28,38 & 27,77 & 27,51 & 27,79 & 27,86 & 28,09 & 27,87 & 28,49 & 27,97 \\
UA & 2011 & 65,45 & 67,48 & 73,23 & 75,92 & 77,36 & 71,14 & 70,00 & 61,98 & 52,23 & 56,63 & 57,56 & 57,99 & 65,58 \\
& 2012 & 81,47 & 84,43 & 84,31 & 83,46 & 78,49 & 79,86 & 77,42 & 72,16 & 70,95 & 72,97 & 77,70 & 77,72 & 78,41 \\
VV & 2011 & 1,88 & 1,56 & 1,21 & 1,00 & 0,97 & 1,11 & 1,31 & 1,70 & 2,30 & 2,81 & 2,89 & 2,93 & 1,81 \\
& 2012 & 2,87 & 2,17 & 2,21 & 2,14 & 2,53 & 2,29 & 2,39 & 2,67 & 3,09 & 3,26 & 3,37 & 3,27 & 2,69 \\
PL & 2011 & 100,58 & 151,38 & 89,92 & 236,98 & 219,44 & 18,54 & 115,06 & 15,24 & 0,00 & 9,14 & 8,13 & 0,00 & 80,37 \\
& 2012 & 28,95 & 67,83 & 28,20 & 33,02 & 0,00 & 26,92 & 13,97 & 0,00 & 0,00 & 0,00 & 0,51 & 0,00 & 16,62 \\
\hline
\end{tabular}

T: Temperatura, UA: Unidade do ar, VV: Velocidade do vento, PL: Pluviosidade.

Xu e Singh(2002),corrobora com os dados obtidos neste trabalho, ao utilizar o modelo de Hargreaves-Samani, obteve tendência de superestimação para ETo em regiões úmidas e subestimação em regiões muito secas e em regiões de velocidade de vento superiores a $4 \mathrm{~ms}^{-1}$. Mesmo assim, o modelo de Hargreaves-Samani, carece de calibração local antes de ser adotado para estimar a ETo em qualquer região distinta (BAUTISTA et al., 2009; FOOLADMAND; HAGHIGHAT, 2007; GAVILÁN et al., 2006).

Verifica-se maior diferença entre os dados obtidos quando contrapostos (Figura 2) os métodos de HS,PM-FAO56 com a pluviosidade. Podendo-se correlacionar com a umidade, uma vez que o HS que apresentou significância $(\mathrm{P}=0,0009599)$ em relação à pluviosidade assim como a EToPM-FAO56 que também apresentou significância $(\mathrm{P}=0,000105)$. Influenciando $\mathrm{O}$ comportamento de ambos os métodos em distintas condições (anos/pluviosidades), o método HS expressou uma variação percentual de $18 \%$ em comparação com PM-FAO56 no ano de 2011 considerado mais úmido, ao atingir pluviometria de 946,42 $\mathrm{mm}_{\mathrm{ano}}{ }^{-1}$.

Figura 2. Comportamento da evapotranspiração anual em relação a pluviosidade anual para os métodos Penman-Monteith-FAO56 (A) e de Hargreaves-Samani (B), o ano 2011 representado pela linha preta (-) e o ano de 2012 representado pela linha cinza (-) em Mossoró, Rio Grande do Norte.
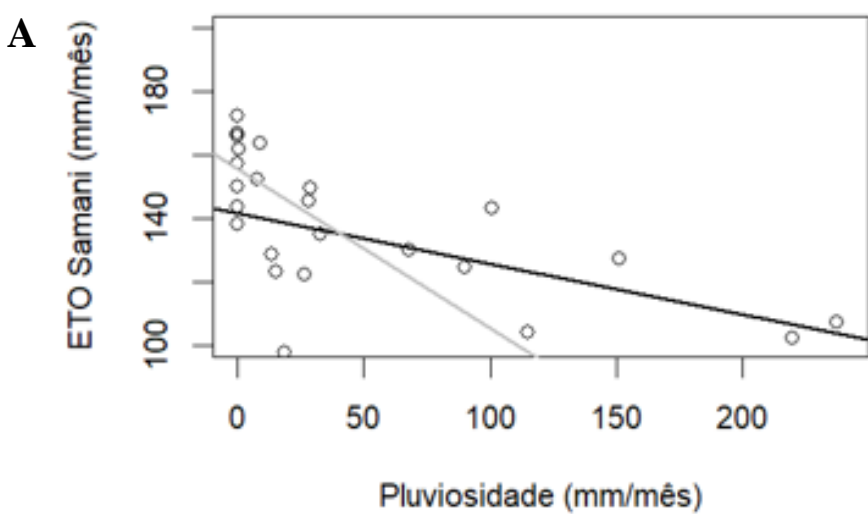

Com uma pluviosidade de 199,41 mm ano, 2012 obteve menor uma variação percentual $(6 \%)$ entre os métodos citados. Isto explicaria a maior proximidade entre os valores encontrados por ambos os métodos analisados no ano de 2012 (Figura 2B), se comparado com 2011 (Figura 2A) que apresentou maior diferença entre os resultados.

Para uma melhor compreensão fez-se uma correlação para efeitos da pluviosidade e velocidade do vento em relação aos métodos distintos de avaliação da ETo. na ETo por HargreavesSamani quando se correlacionando a pluviosidade com velocidade do vento não foi encontrado significância ( $\mathrm{P}=$ 0,49676), mesmo assim houve maior proximidade que quando avaliados para ETo por FAO56 ( $\mathrm{P}=0.865)$ (Figura 3). Ambos as variáveis apresentaram estatisticamente ausência de significância

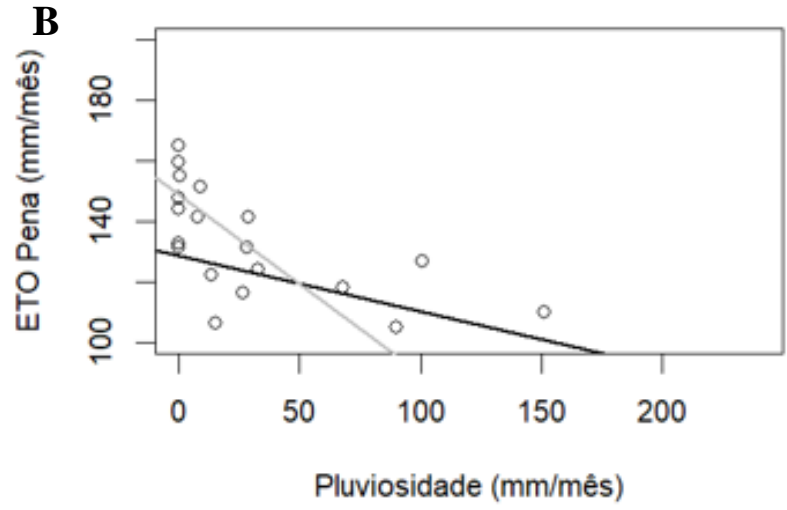

nem a 5 nem a $1 \%$ para o fator pluviosidade quando analisadas de forma isolada embora tenham tido resultados semelhantes.

Ao se analisar o vento este apresentou significância seja a 5 ou $1 \%$ em ambos os métodos de estimativa de ETo HS (P = 0,00314) e PM-FAO56 $\left(\mathrm{P}=6,78 \mathrm{e}^{-10}\right)$, observável nos gráficos abaixo (Figura 3) aEToPM-FAO56 (Figura 3B), exibiu maior concentração de dados na zona de menor pluviosidade e crescimento gradual e acuminado para maiores velocidades de vento, retratando a maior significância apresentada. Já a ETo HS (Figura 3A) apresentou uma maior dispersão dos dados entre menor umidade e maior velocidade do vento, sendo este último mais dominantes em relação aos pontos da ETo. 
Figura 3. ETo Hargreaves-Samani (A) e a ETo PenmanMonteith -FAO56 (B), correlacionando-se dados da pluviosidade com a velocidade do vento ao longo dos anos 2011-2012 em Mossoró, Rio Grande do Norte
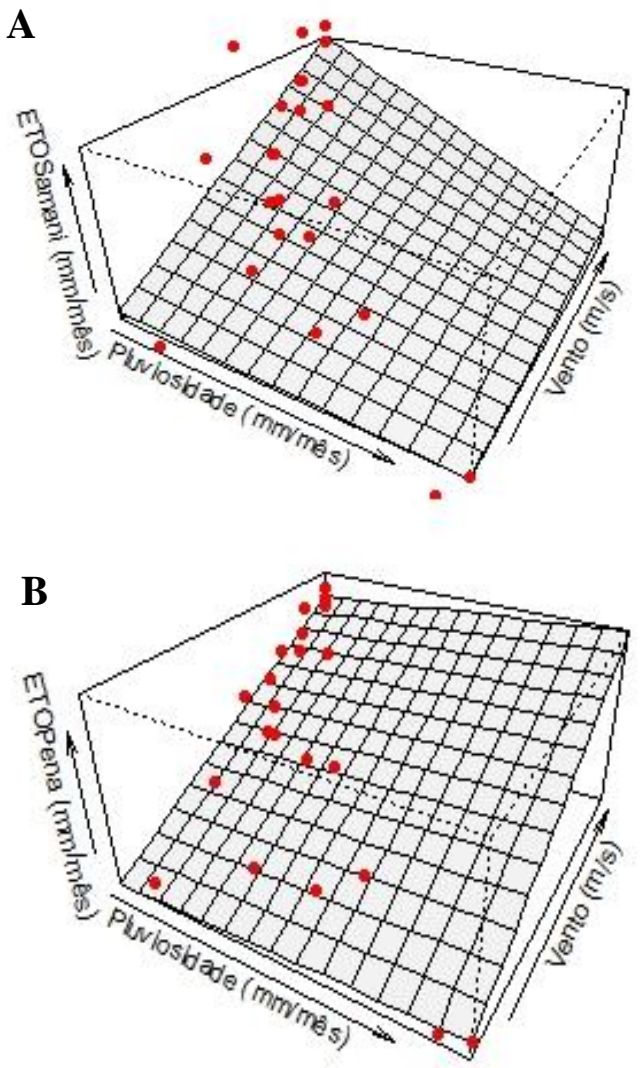

Estudos feitos por Hargreaves-Samani (1982) defendem que o modelo estudado mesmo calibrado não expõe boa adaptação a regiões litorâneas, o que possivelmente justificou ainda mais a disparidade encontrada nos gráficos aqui expostos em relação ao vento assim como sua significância, idêntico ao que Maestre-Valero et al. (2013), observaram ajustando a equação de Hargreaves-Samaniem uma região semiárida da Espanha, manuseando dados de 12 estações automáticas, no litoral o coeficiente ajustado chegou a 0,0034 , à medida que em regiões mais interioranas não ultrapassou 0,0025 .

No México os processos de calibração simultânea e de somente um parâmetro apresentou comportamento também semelhante no litoral, sendo que dessa vez o coeficiente da equação de Hargreaves-Samani não ultrapassou 0,0026 para os meses de abril, maio, outubro e dezembro, em virtude de calibrações feitas mensalmente (BAUTISTA et al., 2009).Por fim, sugere-se para pesquisas posteriores, o ajuste dos modelos e devida análise em escalas de tempo mensais por estas apresentarem menor variação quando comparada a diária.

\section{CONCLUSÕES}

O modelo de Hargreaves-Samani superestimou a equação Penman-Monteith - FAO56 no ano de maior pluviosidade no município de Mossoró (RN), sendo necessário sua calibração para locais com maior influência litorânea.

\section{REFERÊNCIAS}

ALLEN, R. G.; PEREIRA, L. S.; RAES, D.; SMITH, M. Crop evapotranspiration: guidelines for computing crop water requirements. FAO - Food and Agriculture Organization of the United Nations Rome, 1998.

BARBIERI，J. D.; DALLACORT，R.; DANIEL，D. F.; DALCHIAVON, F. C.; FREITAS, P. S. L. Cobertura do solo, evapotranspiração e produtividade do milho safrinha. Cultura Agronômica: Revista de Ciências Agronômicas, v. 29, n.1, p. 7691, 2020. 10.32929/2446-8355.2020v29n1p76-91

BAUTISTA, F.; BAUTISTA, D.; DELGADO-CARRANZA, C. Calibration of the equations of Hargreaves and hornthwaite to estimate the potential evapotranspiration in semi-arid and subhumid tropical climates for regional applications. Atmósfera, v. 22 , n. 4 , p. 331-348, 2009.

CARMO FILHO, F.; ESPÍNOLA SOBRINHO, J.; AMORIM, A. P. Dados meteorológicos de Mossoró (janeiro de 1898 a dezembro de 1986). Mossoró: ESAM/FGD. v. 341, p. 325, 1987.

CONCEIÇÃO, M.A.F. Avaliação de modelos para a estimativa decendial da evapotranspiração de referência. EMBRAPA, 2019.

CAVALCANTE JUNIOR, E. G.; OLIVEIRA, A. D.; ALMEIDA, B. M.; SOBRINHO, J. E. Métodos de estimativa da evapotranspiração de referência para as condições do semiárido Nordestino. Semina: Ciências Agrárias, v. 32, n. 1, p. 1699-1708, 2011. 10.5433/1679-0359.2011v32Suplp1699

DINIZ, M. T. M.; OLIVEIRA, G. P. Proposta de compartimentação em mesoescala para o litoral do Nordeste brasileiro. Revista Brasileira de Geomorfologia, v. 17, n. 3, p. 565-590, 2016. 10.20502/rbg.v17i3.844

FERNANDES, D. S.; HEINEMANN, A. B.; PAZ, R. L. F.; OLIVEIRA AMORIM, A. Calibração regional e local da equação de Hargreaves para estimativa da evapotranspiração de referência. Revista Ciência Agronômica, v. 43, n. 2, p. 246-255, 2012. $10.1590 /$ S1806-66902012000200006

FERNANDES, F. V. R.; NANTES, T. L. G. Comparação de dois diferentes métodos de estimativa de evapotranspiração para diferentes localidades do estado de mato grosso do sul. 2019. Trabalho de Conclusão de Curso (Engenharia Agrícola) Universidade Federal da Grande Dourados, Dourados. 2019.

FERREIRA, L. B.; CUNHA, F. F.; SEDIYAMA, G. C.; CAMPOS, F. B. HS Cal software for the calibration of the Hargreaves-Samani equation. Revista Pesquisa Agropecuária Brasileira, v.54, n. 26, p. 1-5, 2019. 10.1590/S16783921.pab2019.v54.00226

FOOLADMAND, H. R.; HAGHIGHAT, M. Spatial and temporal calibration of Hargreaves equation for calculating monthly ETo based on Penman-Monteith method. Irrigation and Drainage, v. 56, n. 4, p. 439-444, 2007. 10.1002/ird.305 
GAVILÁN, P., LORITE, I. J., TORNERO, S., \& BERENGENA, J. Regional calibration of Hargreaves equation for estimating reference ET in a semi-arid environment. Agricultural Water Management, v. 81, n. 3, p. 257-281, 2006. 10.1016/j.agwat.2005.05.001

HARGREAVES, G. H.; SAMANI, Z. A. Estimating potential evapotranspiration. Journal of Irrigation and Drainage Engeneering, v. 108, p. 225-230, 1982.

HARGREAVES, G. H.; SAMANI, Z. A. Reference crop evapotranspiration from temperature. Applied Engineering in Agriculture, v. 01, n. 02, p. 96-99, 1985.

LIMA JUNIOR, J. C., ARRAES, F. D., DE OLIVEIRA, J. B., NASCIMENTO, A. L., \& DE MACÊDO, K. G. Parametrização da equação de Hargreaves e Samani para estimativa da evapotranspiração de referência no Estado do Ceará, Brasil. Revista Ciência Agronômica, v. 47, n. 3, p. 447-454, 2016. $\underline{10.5935 / 1806-6690.20160054}$

MAESTRE-VALERO, J. F.; MARTÍNEZ-ALVAREZ, V.; GONZÁLEZ-REAL, M. M. Regionalization of the Regionalization of the Hargreaves coefficient to estimate longterm reference evapotranspiration series in SE Spain. Spanish Journal of Agricultural Research, v. 11, n. 4, p. 1137-1152, 2013. $\underline{10.5424 / \mathrm{sjar} / 2013114-3869}$

NICODEM, L. V. S.; MINUZZI, R. B. Estimativa da evapotranspiração de referência diária com base na temperatura do ar para Campos Novos e São José, no estado de Santa Catarina. Agrometeoros, v. 27, n. 2, p. 339-345, 2019. $\underline{10.31062 / \text { agrom.v27i2.26468 }}$

R CORE TEAM. R: A language and environment for statistical computing. 2017.

SENTELHAS, P. C.; GILlESPIE, T. J.; SANTOS, E. A. Evaluation of FAO Penman-Monteith and alternative methods for estimating reference evapotranspiration with missing data in Southern Ontario, Canada. Agricultural Water Management, v. 97, n. 5, p. 635-644, 2010. 10.1016/j.agwat.2009.12.001

SILVA, M. B. P. Análise da evapotranspiração de referência (EToFAO-56) no planalto ocidental paulista. Tese (Doutorado em Agronomia) Faculdade de Ciências Agronômicas da Unesp, Botucatu.2019.

TABARI, H.; GRISMER, M. E.; TRAJKOVIC, S. Comparative analysis of 31 reference evapotranspiration methods under humid conditions. Irrigation Science, v. 31, p.107-117, 2013. $\underline{10.1007 / \mathrm{s} 00271-011-0295-\mathrm{Z}}$

THEPADIA, M.; MARTINEZ, C. J. Regional calibration of solar radiation and reference evapotranspiration estimates with minimal data in Florida. Journal of Irrigation and Drainage Engineering, v. 138, n. 2, p. 111-119, 2012. $\underline{10.1061 /(\text { asce)ir.1943-4774.0000394 }}$
TURCO, J. E. P. Estimativa da evapotranspiração de referência com precisão. Revista Engenharia na Agricultura, V.27, n.2, p.132-141, Viçosa - MG, 2019. 10.13083/reveng.v27i2.876

VENANCIO, L. P.; CUNHA, F. F.; MANTOVANI, E. C.; SEDIYAMA, G. C.; EUGENIO, F. C.; ALEMAN, C. C. Penman-Monteith com dados faltantes e Hargreaves-Samani para estimativa da ETo no estado do Espírito Santo, Brasil. Revista Brasileira de Engenharia Agrícola e Ambiental. v.23, n.3, 2019. 10.1590/1807-1929/agriambi.v23n3p153-159

XU, C. Y.; SINGH, V. P. Cross comparison of empirical equations for calculating potential evapotranspiration with data from Switzerland. Water Research Management, v. 16, n. 3, p. 197-219, 2002. 10.1023/a:1020282515975. 